\title{
Note del CuRAtore e Direttore
}

Questo numero monografico, dal titolo forse ambizioso ma metodologicamente essenziale - Teatro italiano fra testo, performance e scena - mira a contribuire con eccellenti e selezionati saggi al vasto tema del rapporto fra i sopraccitati elementi che, insieme ad altri ma più di altri, danno vita allo spettacolo. Non s'intende certo esaurire un argomento così vasto, in più luoghi affrontato dalla storiografia attraverso varie metodologie. Ciò nonostante, tale articolato e complesso tema, importante e fondamentale per la storia della scena italiana, troverà un sicuro, valido e ulteriore arricchimento attraverso i contributi di questo volume speciale di Quaderni d'italianistica.

\section{ers}

Lo storico del teatro, pur in un vario panorama metodologico, quando si rivolge a reperti del passato vive un'intrinseca, direi necessaria, frustrazione: l'evanescenza della performance. In secoli lontani dalla riproduzione-registrazione audiovisiva il dato della serata, il gesto e la voce dell'attore/attrice, l'occasione stessa della rappresentazione rimangono virtualmente inafferrabili. Di qui l'importanza non solo del testo teatrale, ma anche del contesto che circonda sia il testo stesso, sia lo spettacolo in cui prende vita.

Inoltre, per lo studioso d'opera o di commedia o di interpreti del passato è imprescindibile e necessario rivolgersi a una varia e vasta messe di fonti e contesti a volte apparentemente eterogenei: testi (ma letti come documenti), carte d'archivio, lettere, cronache, memorie, diari di viaggiatori, eventi storici, piante e progetti dei teatri, storie dinastiche, trattati ecc. I settori disciplinari a volte s'intrecciano, dovendo, lo studioso, per osservare il suo oggetto composito (testo, paratesto, attori, messinscena, dedicatari...) rivolgersi alla storia dell'arte, all'architettura, alla storia delle idee, della politica, delle corti, della religione, della letteratura, del viaggio e a molti altri settori disciplinari o "arti." Nel caso dell'opera in musica si aggiungono la musicologia e la storia sia della notazione musicale, sia della coreografia (per la danza e i balli).

A questo proposito, in particolare in ambito italiano tra XVI e XVIII secolo, lo spettacolo poteva basarsi anche su un non-testo, quello dei canovacci della 
commedia dell'Arte in cui la performance dell'attore o dell'attrice (o di gruppi o coppie di attori) diventava il vero testo dello spettacolo spingendo in sottofondo la produzione letteraria a favore di una tecnica d'improvvisazione che si rivolgeva, ai livelli artisticamente più alti, alle figure combinate della retorica — si veda ciò che scrive nel 1699 Andrea Perrucci —, all'uso di formule petrarchesche, a inserti musicali, ma anche, e forse essenzialmente per certa parte del pubblico, al lazzo, alla scenetta comicissima, performativa, basata sul frizzo linguistico e/o l'acrobazia fisica, spesso bagaglio e cifra di un certo attore o di un'attrice — si pensi alla scena della "pazzia d'Isabella" recitata da Isabella Canali Andreini che diventa un intero canovaccio pubblicato da Flaminio Scala col titolo La Pazzia d'Isabella.

Tuttavia non solo di attori professionisti era, e in parte è, fatto il teatro. Anzi, si potrebbe dire che i non professionisti fossero la maggioranza di coloro che si esibivano su vari tipi di scene e in vari tipi di contesti spettacolari. Dai dilettanti cortigiani e dei circoli degli umanisti agli studenti e ai giovani membri delle confraternite o delle compagnie della calza e così via. A molte altre realtà, spesso private e domestiche, si affidava il teatro, che era agito nelle scuole, nei collegi dei gesuiti (dalla metà del XVI sec.), nelle confraternite, nei palazzi reali o nobiliari o anche in benestanti dimore borghesi, sui sagrati delle chiese e nelle chiese stesse - si ricordino le grandi sacre rappresentazioni di stile fiorentino allestite da Brunelleschi nella Firenze del Quattrocento e in occasione del concilio del 1439, evento fondante per la cultura fiorentina rinascimentale (e non solo) — ma anche nelle piazze, sulle panche dei montipanca, sui piccoli palcoscenici dei venditori di specifici.

Appunto, il luogo dello spettacolo - luogo teatrale, si dovrebbe dire, seguendo un'acquisita differenziazione che distingue tra luogo teatrale ed edificio teatrale - , che da indefinito diviene sempre più definito e specifico, oltre che via via incluso nel tessuto urbanistico maggiore delle città, fino a giungere a una vera proliferazione di teatri e teatrini nel Settecento (in città, paesi e financo borghi, ville). Il secolo di Goldoni e Metastasio può ben essere definito un'epoca di teatro e rivoluzioni, borghesi ma anche arcadiche e massoniche.

Il testo stesso prende anche altre strade, altre forme e si avvicina a una "intenzione" più moderna, non solo con la riforma del grande autore veneto della metà del XVIII secolo, ma anche attraverso una maggiore, più incisiva, più dirigista presenza del drammaturgo nei testi stessi, attraverso didascalie e indicazioni sceniche. 
Un teatro, dunque, che si divide non solo tra professionismo, strategie autopromozionali — mirate anche all'avanzamento sociale — e dilettantismo di vario livello, ma anche in generi, ognuno dei quali è in relativa o accesa competizione con gli altri: il teatro comico in prosa con quello serio, il teatro improvviso (la commedia dell'Arte, sostanzialmente) con il teatro "premeditato," il teatro musicale con l'opera seria da un lato e quella buffa dall'altro rivali tra loro, e l'opera buffa era a sua volta in concorrenza con il teatro comico coevo, da cui era "attratta" ma che voleva tenere lontano per motivi di prestigio di "casta." Si assiste anche, e sempre più frequentemente, al formarsi di compagnie per tutte le stagioni o $\mathrm{i}$ generi e che offrivano tragedie, commedie, opere buffe e/o intermezzi musicali comici all'interno di un vorticoso aumento sia dell'offerta, sia della domanda di spettacolo.

La concorrenzialità tra generi e professionisti, se vista da vertici troppo alti, si potrebbe "rinchiudere," valutandola, in considerazioni solo culturali e artistiche. Invece, dalla metà del XVI sec., con la vendita del teatro, da quello dell'Arte ai primi teatri d'opera a pagamento (a Venezia nella prima metà del Seicento), il fenomeno diventa un vasto mercato che coinvolge anche l'editoria e altri settori produttivi, e in cui testi, professionisti, autori, attori, impresari, musicisti, scenografi, falegnami, costumisti e i teatri stessi sono in "concorrenza" tra loro per il prestigio, per la sopravvivenza, per la "mercatura" dello spettacolo. La combinazione di testo e professionismo teatrale, associata a un'evoluzione dei generi (l'opera buffa e seria), e a una proliferazione degli edifici teatrali, influenzerà, assieme al cambiare delle stagioni e dei gusti culturali, il mutare dei generi stessi.

Per corti aristocratiche, teatri, stanze per le commedie, attraverso l'attività di intellettuali e professionisti, o nelle pagine dei trattati e dei testi si articola questo numero monografico che spazia dal rinascimento fiorentino e napoletano al Novecento italiano e di nuovo napoletano, passando per il prezioso Settecento musicale e in prosa. Si noterà nei saggi uno "sbilanciamento" sia a favore del Cinquecento e del Settecento, sia a favore di Napoli e Firenze, con l'inclusione però anche di Venezia. Uno squilibrio "tendenzioso" e del tutto intenzionale. Infatti, un numero più concentrato sulla varietà delle piazze teatrali avrebbe richiesto uno spazio molto più ampio di quello a disposizione. Da questo deriva la scelta di eleggere certi aspetti e certe tradizioni locali nella selezione dei contributi. Gli interessi del curatore, che si articolano, per l'appunto, fra teatro italiano del XV, XVI e XVIII secolo, segnatamente tra Napoli e Firenze, hanno sicuramente influenzato la fisionomia storica di questo volume. 
Delle importanti e interessantissime sperimentazioni quattrocentesche napoletane d'ambito aragonese, con il sovrapporsi di complesse filiere culturali, tratta l'esteso e ricco saggio di Francesca Bortoletti. La cultura spettacolare durante il dominio degli Aragonesi non è stata oggetto di numerosi e validi studi, come meriterebbe, forse anche per le caratteristiche di "città-stato teatrale" di Napoli, slegata dallo scintillante e "sperimentale" circuito delle corti del centro-nord e della Roma papale. Il presente contributo arricchisce sensibilmente la comprensione di questo particolare e rilevante fenomeno del teatro del rinascimento che negli ultimi anni ha visto l'apporto di importanti contributi, incluso quello di Bortoletti. Fu un mondo, quello del teatro napoletano rinascimentale, che coinvolse figure cardine dello spettacolo come Francesco de' Nobili detto Cherea, e famiglie centrali per la committenza e la propagazione di testi e idee del teatro umanista come i Sanseverino.

Michel Plaisance si occupa di uno dei testi più affascinanti del primo Cinquecento fiorentino, anche a causa del destino dell'autore: L'Aridosia di Lorenzino de' Medici (Lorenzo di Pierfrancesco de' Medici), il "Lorenzaccio," il Bruto del Rinascimento, che affascinò i romantici ma anche Carmelo Bene e che assassinò il duca Alessandro de' Medici, per essere poi ucciso dai sicari di Cosimo I. Lo studioso francese presenta un'acquisizione importante per la comprensione di questa perla della drammaturgia fiorentina, e italiana, dei primi anni del principato mediceo: un manoscritto della biblioteca Riccardiana di Firenze che Plaisance utilizza per chiarire il vero "senso" del testo che, come è nello stile metodologico dello studioso di Sorbonne Nouvelle-Paris III, viene illustrato anche attraverso una serrata contestualizzazione degli obiettivi politici e la ricognizione di ricchissime filiere culturali e testuali.

Konrad Eisenbichler, studioso a cui questa rivista deve molto, presenta e discute, invece, un grande drammaturgo della Firenze e dell'Italia del Cinquecento: il notaio Giovan Maria Cecchi, il più prolifico tra gli autori dei suoi anni e del suo ambiente. In lui la compenetrazione utilitaristica, e artistica, dei generi assume aspetti emblematici sia per la perizia con cui essa è condotta, sia per il contesto, come esemplifica Eisenbichler (massimo esegeta di Cecchi), sociale e performativo fiorentino. Lanalisi che ne risulta mette insieme, di questo importante rappresentante della cultura teatrale del XVI secolo, il testo con gli aspetti performativi, "comici" e sociali, intersecando realtà teatrali con altri aspetti del tempo, che vanno dal teatro nelle confraternite alle commistioni tra testi "rinascimentali" e teatro 
sacro, ma anche tra commedia dell'Arte e teatro "colto," con esiti analitici inattesi e importanti.

Rosalind Kerr, esperta di attrici e teatro barocco italiani (in particolare la commedia dell'Arte) ci presenta le "dive" della scena italiana tardo-rinascimentale e barocca. Attrici, o meglio sarebbe dire donne, che usarono le loro capacità intellettuali e letterarie per crearsi una persona oltre quella della scena - e oltre quella oscena che le marchiava socialmente - che potesse elevarle nel consesso delle arti e della società. Strategie testuali e culturali mirate e brillanti da vere dive ante litteram che molto hanno fatto per l'emancipazione della figura dell'attrice, della professione teatrale al femminile, e della donna in generale.

Il saggio di Anthony DelDonna, eccellente studioso del mondo musicale napoletano del Settecento (segnatamente della seconda metà del secolo), esplora un particolare e rilevante aspetto di una cultura della "riforma" che dal nord influenza, al di là dei noti e oramai studiatissimi esiti metastasiani locali, l'opera seria. Mettendo al centro della sua analisi un importante trattato di Antonio Planelli (Dell'opera in musica, 1772), DelDonna evidenzia lo svilupparsi di particolari forme di opera sacra, o, meglio, teatro musicale sacro che ebbero nella Napoli borbonica del secondo Settecento risultati e creazioni singolarissimi e importanti. Dalla corte aragonese il passaggio alla scintillante, mercantile, e artistica al tempo stesso, vita teatrale del secolo d'oro per la scena musicale napoletana rappresenta un'importante ed essenziale panoramica su una delle grandi capitali dello spettacolo europeo. Il saggio si conclude con un'attenta analisi dell'azione sacra della Quaresima, un genere particolarmente napoletano, legato al borbonico teatro di San Carlo, che colmava gli spazi vuoti lasciati nella programmazione stagionale a causa delle proibizioni teatrali durante la quaresima con una tipologia particolarissima di drammaturgia sacro-musicale-devozionale.

Roberta Turchi ci conduce verso un aspetto essenziale dell'uso di vari strumenti oltre quelli testuali per l'analisi della drammaturgia goldoniana: le illustrazioni di Pietro Antonio Novelli alle edizioni Pasquali e Zatta dell'opera dell'avvocato veneziano. Un'altra filiera, nel composito scacchiere metodologico a cui si faceva riferimento, che implica un avvicinarsi — impossibile tanto quanto affascinante - a quell'inafferrabile oggetto di studio che è la serata teatrale, lo spettacolo del passato, l'attore, la messinscena, la recitazione, lo stile. La studiosa sostiene come lo sguardo di Novelli si riveli analogo a quello di critico, tanto da portarci, appunto, verso una maggiore comprensione del teatro di Goldoni, 
di cui l'autrice analizza, alla luce delle illustrazioni e di altri elementi, un'opera fondamentale: La Trilogia della villeggiatura.

Questa raccolta di saggi si chiude con un balzo sul miglior Novecento italiano. Di nuovo Napoli, città che più di altre, o forse come nessun'altra, mantiene, nella tradizione delle famiglie teatrali (di buffi nell'opera o di attori nel teatro comico), un forte legame con il passato, insieme a un'intrinseca ed endemica sovrapposizione di generi (musica, teatro, letteratura dialettale). Pasquale Sabbatino analizza i rapporti di uno dei più grandi esponenti di questa peculiarità performativa locale, Peppino De Filippo, con Luigi Pirandello per gli adattamenti teatrali fatti da Peppino da testi del maggior drammaturgo italiano del XX secolo. Siamo così nel vivo del rapporto fra testo e attore che, nel caso napoletano, implica ulteriori convergenze come quelle che riguardano lingua e dialetto. La tradizione "alta" di Pirandello (pur sempre, però, legatissima al teatro vivo degli attori), si lega a quella dell'attore di tradizione famigliare e al suo utilizzo del testo. Una serrata ricognizione di edizioni e scambi epistolari illumina di nuova e importante luce i rapporti tra il più particolare dei De Filippo, Peppino, e il più innovativo dei drammaturghi italiani del secolo scorso.

\section{es}

Con questo volume speciale sul teatro si conclude il mio mandato quadriennale come direttore/editor di Quaderni d'italianistica. Un lavoro in cui sono stato affiancato dalla dr.ssa Patrizia Bettella, che ringrazio per la sua grande competenza e la preziosa amicizia, e dal prof. Konrad Eisenbichler, che ringrazio per il grandissimo contributo che ha continuamente dato a questa pubblicazione. Il mio e nostro lavoro è stato reso possibile da un eccellente Editorial Board che ha permesso l'uscita regolare di due numeri l'anno (a stampa e online) nel corso di quattro anni. Intendo dunque ringraziare nella maniera più affettuosa e riconoscente la dr.ssa Anne Urbancic e la dr.ssa Paola Basile (book review editors); la dr.ssa Gessica De Angelis e il dr. Gabriele Scardellato (promotion \& developement); e, infine ma non ultima, la dr.ssa Manuela Scarci, Business manager della rivista. Intendo, e posso dire intendiamo, ringraziare anche il nostro prestigioso Advisory Board per il contributo dato alla nostra pubblicazione. Desideriamo anche ringraziare la Canadian Society for Italian Studies (CSIS), editore della rivista, per il validissimo sostegno lungo questo intenso percorso. Un ringraziamento particolarmente sentito, infine, ai molti studiosi e alle molte studiose che con i loro articoli o le 
loro valutazioni per il peer-review hanno reso possibile l'alto livello qualitativo di Quaderni d'italianistica. Grazie a tutti.

Gianni Cicali, Georgetown University 
